\title{
A second post-AGB nebula that contains gas in rotation and in expansion: ALMA maps of IW Carinae
}

\author{
V. Bujarrabal ${ }^{1}$, A. Castro-Carrizo ${ }^{2}$, J. Alcolea ${ }^{3}$, H. Van Winckel $^{4}$, C. Sánchez Contreras ${ }^{5}$, and M. Santander-García ${ }^{3,6}$ \\ 1 Observatorio Astronómico Nacional (OAN-IGN), Apartado 112, 28803 Alcalá de Henares, Spain \\ e-mail: v.bujarrabal@oan.es \\ 2 Institut de Radioastronomie Millimétrique, 300 rue de la Piscine, 38406 Saint-Martin d'Hères, France \\ 3 Observatorio Astronómico Nacional (OAN-IGN), C/ Alfonso XII, 3, 28014 Madrid, Spain \\ ${ }^{4}$ Instituut voor Sterrenkunde, K.U.Leuven, Celestijnenlaan 200B, 3001 Leuven, Belgium \\ ${ }^{5}$ Centro de Astrobiología (CSIC-INTA), Ctra. M-108, km. 4, 28850 Torrejón de Ardoz, Madrid, Spain \\ 6 Instituto de Ciencia de Materiales de Madrid (CSIC), Calle Sor Juana Inés de la Cruz 3, 28049 Cantoblanco, Madrid, Spain
}

Received 15 July 2016 / Accepted 2 December 2016

\section{ABSTRACT}

\begin{abstract}
Aims. We aim to study the presence of both rotation and expansion in post-AGB nebulae, in particular around IW Car, a binary post-AGB star that was suspected to be surrounded by a Keplerian disk.

Methods. We obtained high-quality ALMA observations of ${ }^{12} \mathrm{CO}$ and ${ }^{13} \mathrm{CO} J=3-2$ lines in IW Car. The maps were analyzed by means of a simplified model of $\mathrm{CO}$ emission, based on those used for similar objects.

Results. Our observations clearly show the presence of gas components in rotation, in an equatorial disk, and expansion, which shows an hourglass-like structure with a symmetry axis perpendicular to the rotation plane and is probably formed of material extracted from the disk. Our modeling can reproduce the observations and shows moderate uncertainties. The rotation velocity corresponds to a central stellar mass of approximately $1 M_{\odot}$. We also derive the total mass of the molecule-rich nebula, found to be of $\sim 4 \times 10^{-3} M_{\odot}$; the outflow is approximately eight times less massive than the disk. From the kinematical age of the outflow and the mass values derived for both components, we infer a (future) lifetime of the disk of approximately 5000-10 $000 \mathrm{yr}$.
\end{abstract}

Key words. stars: AGB and post-AGB - circumstellar matter - radio lines: stars - planetary nebulae: individual: IW Car

\section{Introduction}

Keplerian disks around post-AGB stars have been proven to be very elusive. Gas in rotation has been directly observed in only two nebulae to date, the Red Rectangle and AC Her (Bujarrabal et al. 2013b, 2015), by means of interferometric mm-wave maps of CO lines. The Red Rectangle and AC Her belong to a class of binary post-AGB stars with low-mass nebulae and with several independent lines of evidence of disks (e.g., Van Winckel 2003; de Ruyter et al. 2006; Gezer et al. 2015; Bujarrabal et al. 2013a). They are characterized, in particular, by spectral energy distributions (SEDs) with a NIR excess that indicates hot dust close to the stellar system. Single-dish observations of ${ }^{12} \mathrm{CO}$ and ${ }^{13} \mathrm{CO} \mathrm{mm}$-wave emission in these post-AGB stars systematically yielded characteristic line profiles, which are strikingly similar to those of the Red Rectangle and AC Her and to those expected to be emitted by relatively extended Keplerian disks (Bujarrabal et al. 2013a). A slowly expanding component was also proposed to be present in this class of objects from those CO data. ALMA maps of CO lines in the Red Rectangle indeed show a bipolar low-velocity outflow (Bujarrabal et al. 2013b, 2016), very probably formed of gas extracted from the disk and containing a mass approximately ten times smaller. Such a component was also confirmed from CO maps of another of these NIR-excess post-AGBs, 89 Her (Bujarrabal et al. 2007). Rotation was not actually resolved in $89 \mathrm{Her}$, but a small disk could be confined to the prominent central condensation. In this source (and probably in others observed in single-dish), the contribution to the total emission of the outflow is dominant and the outflow contains a mass at least comparable to that of the compact disk. On the other hand, no sign of outflow was found in the maps of AC Her, in which the expanding gas is probably very diffuse.

The evolution of these objects is not well known (e.g., De Marco 2014) and could be very different from that of highmass (pre)planetary nebulae. However, both kinds of sources share remarkable properties, such as dominant axial symmetry, which has been proposed to be associated with rotating disks (e.g., Sánchez Contreras et al. 2002; Soker 2001; Balick $\&$ Franck 2002). Therefore, the study of our objects, the only post-AGB ones in which disks are detected, could be relevant to understand the formation of post-AGB nebulae in general. Moreover, disks are observed in binary post-AGB stars that, surprisingly, show orbits of insufficient size to accommodate an AGB star (Gezer et al. 2015; Van Winckel et al. 2009). In the best-studied nebula, the Red Rectangle (Bujarrabal et al. 2013b, 2016), the total angular momentum of the disk is not negligible and, provided that all momentum comes from the binary system, would imply a significant decrease of the distance between the stars. Better constraints on the structure and evolution of the disks are therefore imperative to studying the orbital evolution of these systems and their late evolution.

We present ALMA maps of IW Car that clearly show both rotating and expanding gas. A simplified model is compared with the observations, allowing us to estimate the main nebular parameters. IW Car is an RV Tau variable (Kiss et al. 2007) that belongs to the class of NIR-excess post-AGB stars mentioned 


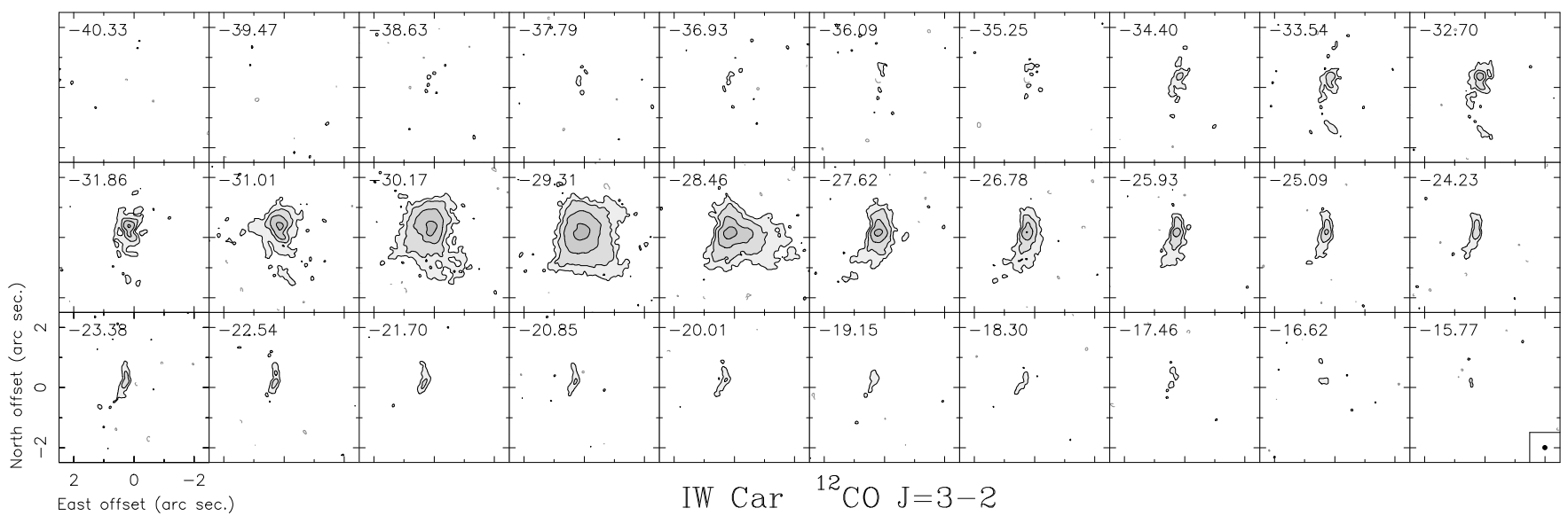

Fig. 1. ALMA maps per velocity channel of ${ }^{12} \mathrm{CO} J=3-2$ emission in IW Car. The continuum has been subtracted to better show the distribution of the weak line. The contour spacing is logarithmic: $\pm 5,15,45$, and $135 \mathrm{mJy} /$ beam (equivalent to $\pm 1.8,5.1,15.4$, 46.2 K, in Rayleigh-Jeans equivalent temperature). The LSR velocities are indicated in each panel (upper-left corner) and the insert in the last panel shows the beam width.

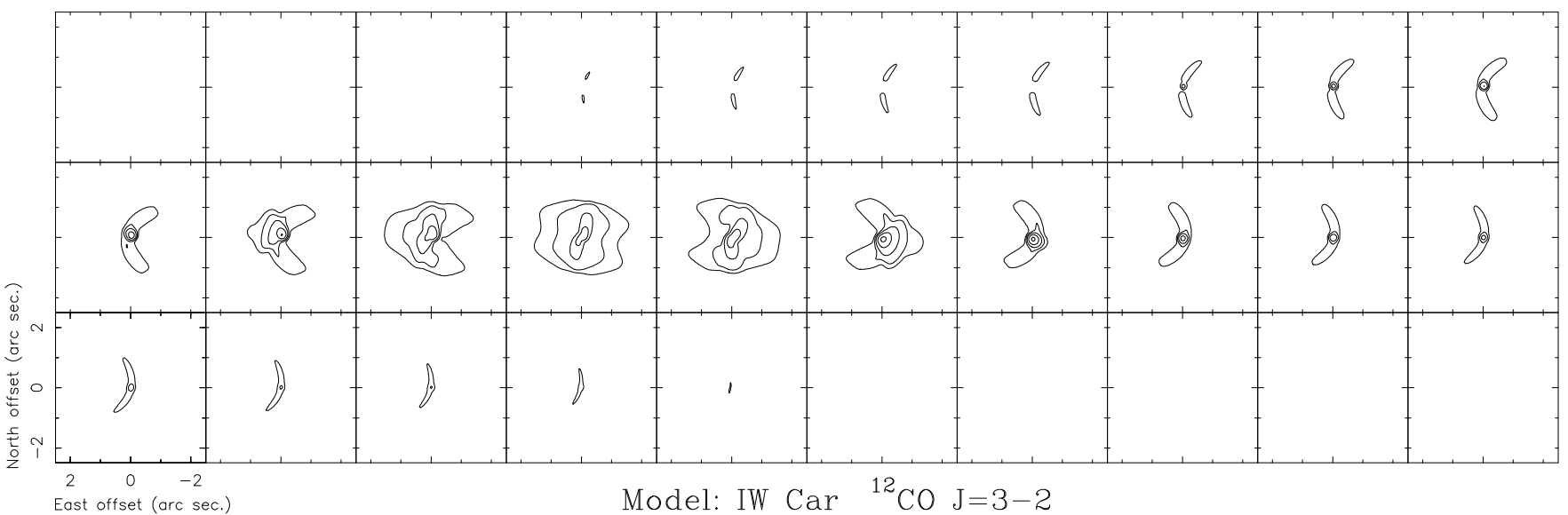

Fig. 2. Predictions of our nebula model for ${ }^{12} \mathrm{CO} J=3-2$ emission in IW Car (Sect. 3.1). To be compared with Fig. 1; all scales and contours are the same as in that figure.

above (Giridhar et al. 1994; De Ruyter et al. 2006; Bujarrabal et al. 2013a). A characteristic size of the hot-dust component of $\sim 5 \times 10^{15} \mathrm{~cm}$ has been derived from model fitting of the SED, although the nebular shape has not been well studied. IW Car is probably a double stellar system (with poorly known orbital parameters) and shows a particularly high atmospheric depletion. Following those authors we adopt a distance of $1 \mathrm{kpc}$ for this source, but we stress that this value is uncertain.

\section{Observations}

We present maps of IW Car in the ${ }^{12} \mathrm{CO}$ and ${ }^{13} \mathrm{CO} J=3-2$ lines, $\lambda=0.8 \mathrm{~mm}$, obtained with ALMA, receiver band 7. Four tracks were consecutively observed on July 26, 2015, with an array configuration of 42 antennas providing baselines between 12 and $1572 \mathrm{~m}$. In total, $110 \mathrm{~min}$ of acquisitions were obtained on source. The data were first calibrated with the CASA software package. The quasars J0522-3627 and J1058+0133 were used to calibrate the bandpass, and J0842-6053 to calibrate gains. J0538-440 and J1107-448 were set as absolute flux references (with $0.91 \mathrm{Jy}$ and $0.43 \mathrm{Jy}$ at $345.8 \mathrm{GHz}$ respectively). In addition, phase self-calibration was later performed using the compact continuum source as a reference with the GILDAS software package, which was also used in the following data analysis. Image deconvolution was performed by using natural weighting, leading to a resolution (HPBW) of approximately 0 , $17 \times 0$." 17 .
The backends were set to achieve a spectral resolution of approximately $0.21 \mathrm{~km} \mathrm{~s}^{-1}$, which was degraded for our final maps because of the poor $\mathrm{S} / \mathrm{N}$ at high velocities, to a resolution of $0.85 \mathrm{~km} \mathrm{~s}^{-1}$ for ${ }^{12} \mathrm{CO} \mathrm{J}=3-2$ (which shows emission at larger velocities) and of $0.66 \mathrm{~km} \mathrm{~s}^{-1}$ for ${ }^{13} \mathrm{CO} J=3-2$. By comparison with the APEX single-dish profile (Bujarrabal et al. 2013b), we conclude that a small fraction of $\sim 25 \%$ of the flux has been filtered out in the maps of ${ }^{12} \mathrm{CO} J=3-2$. The percentage of lost flux could be higher in the line wings, at $\pm 3-4 \mathrm{~km} \mathrm{~s}^{-1}$ from the velocity center, but remains moderate throughout, $\$ 50 \%$. Dust continuum emission was detected, with total flux $\sim 0.2 \mathrm{Jy}$, and barely resolved, with a tentative (deconvolved) size $\sim 0$.' 1 .

\section{Results}

In Fig. 1, we show our ALMA maps per velocity channel of the ${ }^{12} \mathrm{CO} J=3-2$ emission (in Fig. 2 we show the corresponding predictions of our model, see below). We note the clear detection of an hourglass-like structure, whose symmetry axis direction, projected in the plane of the sky (position angle), is $\mathrm{PA} \sim 75^{\circ}$. The structure is comparable to that found in the similar object 89 Her (Sect. 1; but smaller in angular units). The strikingly similar distributions of both structures strongly suggest that these lobes are expanding, as confirmed by the model we describe below. Figure 3 shows our maps of ${ }^{13} \mathrm{CO} J=3-2$. A hint of the lobes can be seen, but their intensity is certainly lower than for 


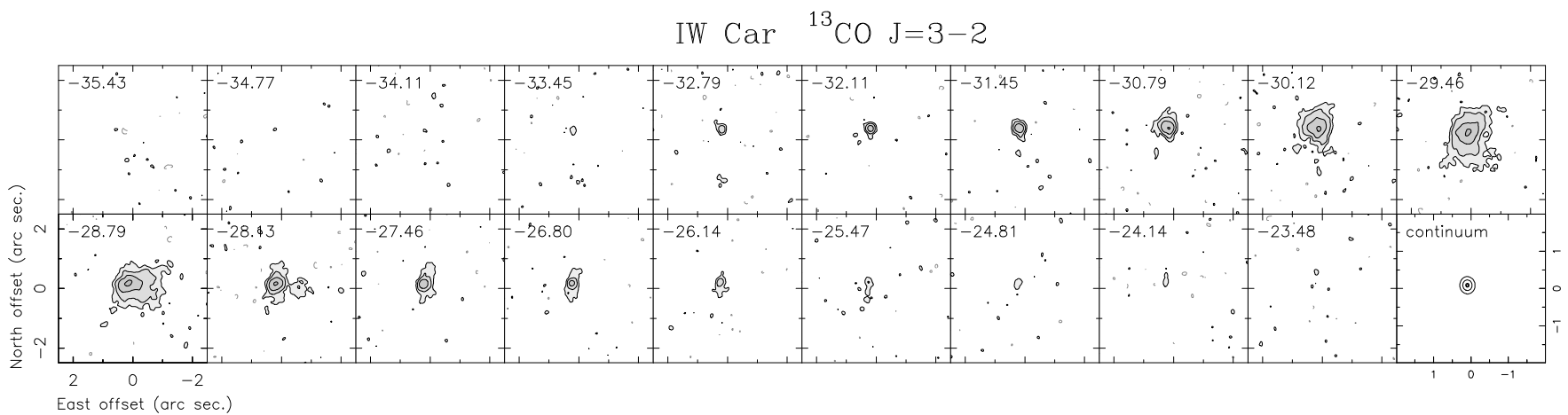

Fig. 3. ALMA maps per velocity channel of ${ }^{13} \mathrm{CO} J=3-2$ emission in IW Car. The continuum has also been subtracted in this figure. The contours are the same as in Fig. 1: $\pm 5,15,45$, and $135 \mathrm{mJy} /$ beam (equivalent to $\pm 1.7,5.3,16.9,47.8 \mathrm{~K}$ ). The LSR velocities are indicated in each panel (note that the velocity resolution is higher than in Fig 1). The last panel shows the continuum emission, contours: 5, 15, 45, and 135 mJy/beam.

${ }^{12} \mathrm{CO} J=3-2$ and the central condensation largely dominates. A lower spectral resolution is used for ${ }^{12} \mathrm{CO} J=3-2$ because it shows the wide and weak emission from the outflow, better seen in wider channels.

Rotation is also clearly detected in our maps of IW Car. Figures 4 and 5 show position-velocity diagrams along the direction perpendicular to the outflow axis, which corresponds to the equatorial plane, at $-15^{\circ}$. The Keplerian pattern is very obvious in both ${ }^{12} \mathrm{CO}$ and ${ }^{13} \mathrm{CO}$ diagrams. We recall that 89 Her shows expanding lobes similar to those found here, but no rotation was detected in it, although Bujarrabal et al. (2007) argued in favor of the presence of rotation within the central unresolved clump.

Among the direct observational facts, we underline the particularly high peak brightness found in the maps, of almost $100 \mathrm{~K}$. These high values, very similar to the high brightness found in the Red Rectangle (Bujarrabal et al. 2013b, 2016), and the comparable peak intensities found for ${ }^{12} \mathrm{CO}$ and ${ }^{13} \mathrm{CO} J=3-2$ suggest that the emission of the central parts of the disk is optically thick (mostly for ${ }^{12} \mathrm{CO}$ ) and that the temperatures in IW Car should be high and similar to those deduced for the Red Rectangle, $>100 \mathrm{~K}$ in central regions. We also point out the presence of blueshifted absorption of the relatively intense emission of central regions by outer and cooler expanding gas approaching us, as shown by the low negative contours that appear at approximately $-35 \mathrm{~km} \mathrm{~s}^{-1}$ in Figs. 1 and 4; this effect is expected in expanding nebulae and is in fact also found in our modeling, see further discussion in Appendix A.3.

\subsection{Simple modeling of our ALMA maps}

The $\mathrm{S} / \mathrm{N}$ and angular resolution of our data are moderate and, moreover, only maps of ${ }^{12} \mathrm{CO}$ and ${ }^{13} \mathrm{CO} J=3-2$ are available. We also lack information on the nebula in general, including only single-dish observations of ${ }^{12} \mathrm{CO} J=2-1$ and $J=3-2$. Under these conditions, very detailed models, as those developed for the Red Rectangle, are not sensible and we only perform simplified modeling. See the predictions of our model in Fig. 2 for ${ }^{12} \mathrm{CO} J=3-2$ and a general description of the model nebula in Fig. 6; the observed maps are reasonably reproduced. See further discussion and details on our modeling in Appendix A.

In view of the similar outflows and disks found in IW Car and in 89 Her and the Red Rectangle (Sect. 1), we base our modeling on the properties we previously derived for those objects. The width of the disk is not easy to determine from the data because of the relatively low angular resolution, we adopt a shape similar to that found in the Red Rectangle. In our

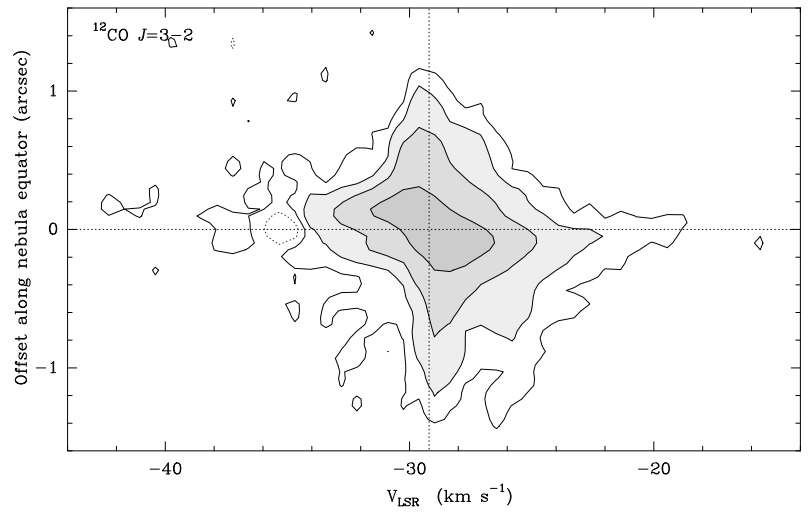

Fig. 4. Position-velocity diagrams from our ALMA maps of ${ }^{12} \mathrm{CO} J=3-2$ in IW Car along the direction PA $=-15^{\circ}$. Contours and the rest of the imaging parameters are the same as in the channel maps. The pointed lines show approximate centroids in velocity and position.

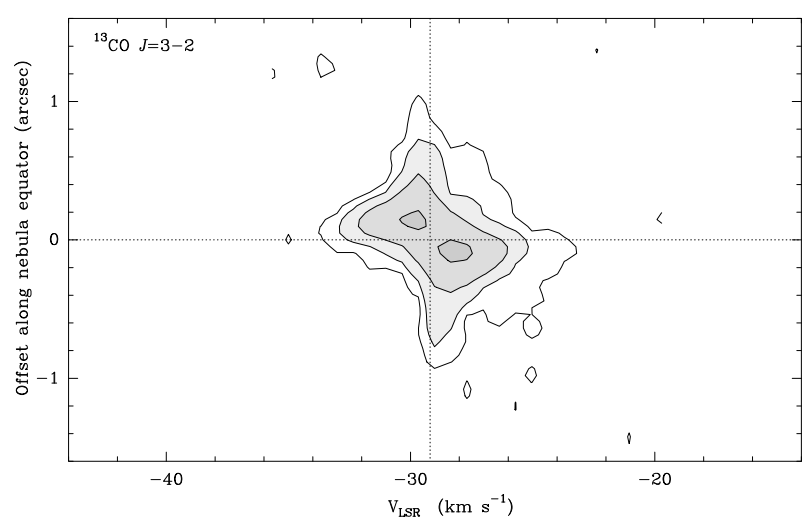

Fig. 5. As in Fig. 1 but for ${ }^{13} \mathrm{CO} J=3-2$.

best-fit model, the expansion velocity is radial and its modulus is assumed to vary with the distance to the equator and to the axis (see Appendix A.1). The disk rotation is Keplerian with $V\left(2 \times 10^{15} \mathrm{~cm}\right)=2.5 \mathrm{~km} \mathrm{~s}^{-1}$, which corresponds to a central stellar mass of $\sim 1 M_{\odot}$; this mass value is reasonable for a low-mass post-AGB double system, though it is smaller than that found in the Red Rectangle $\left(\sim 1.7 M_{\odot}\right)$. The velocity value is uncertain by approximately $\pm 25 \%$ (also taking into account the uncertainty in the inclination, see below). The uncertainty of the deduced central mass is $1_{-0.4}^{+0.6} M_{\odot}$. The axis inclination with respect to the plane of the sky is poorly determined; it cannot be either too 


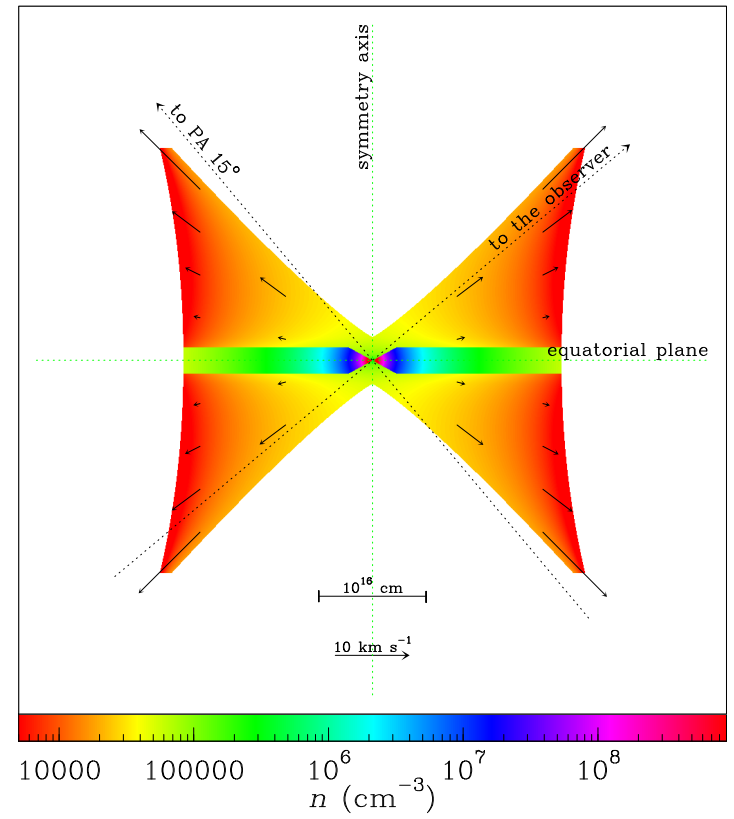

Fig. 6. Structure and distributions of the velocity and density (in color) of our best-fit model for the disk and outflow. Only the velocity of the outflow is shown. The inclination of the disk with respect to the line of sight is shown by the red-dashed line.

large or too small in order to explain the easy detection of both expansion and rotation. We adopt a value of $\sim 45^{\circ}\left( \pm 15^{\circ}\right)$.

We assume LTE populations for the involved rotational levels. This is a reasonable assumption for low- $J$ CO transitions in the dense material expected in our sources, $n>10^{4} \mathrm{~cm}$, since their Einstein coefficients are more than ten times smaller than the typical collisional rates; see further discussion in Bujarrabal et al. (2013b, 2016). The use of LTE may introduce some uncertainties (Appendix A.2), but it simplifies significantly the calculations and provide an easier interpretation of the fitting parameters.

We adopt a relative abundance $X\left({ }^{13} \mathrm{CO}\right) \sim 2 \times 10^{-5}$, to ease the comparison with previous results on this object (Bujarrabal et al. 2013a). In order to match the relatively low ${ }^{12} \mathrm{CO} /{ }^{13} \mathrm{CO}$ intensity ratio, we need a low abundance ratio, we deduce $X\left({ }^{12} \mathrm{CO}\right) \sim 10^{-4}$; similar low ratios are often found in similar objects. ${ }^{13} \mathrm{CO}^{\sim} J=3-2$ is weaker than ${ }^{12} \mathrm{CO} J=3-2$, confirming that the ${ }^{13} \mathrm{CO}$ emission is not very opaque. Our models yield values of $\tau\left({ }^{13} \mathrm{CO} J=3-2\right)$ smaller than $\sim 0.3$ in most of the disk and significantly smaller in the outflow (see Appendix A.2). The density and temperature ( $n$ and $T$ ) are assumed to depend solely on the distance to the center and the equator with potential laws. See more details in the appendix; the density distribution is shown in Fig. 6. The total mass derived from our fitting is $4 \times 10^{-3} M_{\odot}$, very similar to the value found by Bujarrabal et al. (2013a) from an analysis based on very preliminary information on the properties of the nebula and only ${ }^{12} \mathrm{CO}$ single-dish profiles. The mass of the outflow is approximately 8 times smaller than that of the disk.

\section{Summary and conclusions}

We present high-quality ALMA observations of ${ }^{12} \mathrm{CO}$ and ${ }^{13} \mathrm{CO} J=3-2$ line emission in IW Car. IW Car belongs to a class of binary post-AGB stars that show properties suggesting the presence of Keplerian disks around them (Sect. 1). Maps per velocity channel, Figs. 1 and 3, show an hourglass-like nebula in expansion, with an axis oriented along position angle $\mathrm{PA} \sim 75^{\circ}$. This structure is very similar to that detected in a similar object,
89 Her (Bujarrabal et al. 2007). In the position-velocity diagrams along the perpendicular direction, for PA $=-15^{\circ}$, Figs. 4 and 5, we easily recognize the presence of a disk in Keplerian rotation, probably in the equator of the nebula. Similar rotating disks had been well identified before in only two post-AGB nebulae, the Red Rectangle and AC Her, which also belong to the same class of binary stars. The expected presence of both rotating and expanding gas had been well confirmed before in only the best studied of these objects, the Red Rectangle, where the outflow was shown to probably be extracted from the disk (Sect. 1).

Our modeling of the observations is simple but is able to reproduce them and confirms our conclusions above (Sect. 3.1; Figs. 2 and 6). We find the Keplerian rotation to be compatible with a central stellar mass of $\sim 1 M_{\odot}$, reasonable for a post-AGB (double) star of this kind. High temperatures of approximately $100 \mathrm{~K}$ were typically found in the nebula. The total nebular mass is found to be $\sim 4 \times 10^{-3} M_{\odot}$, with the mass of the outflow being approximately eight times smaller than that of the disk. These mass values are $\sim 2.5$ times lower than those found in the Red Rectangle, but the disk/outflow mass ratios are very similar in both nebulae and the general structure and dynamics are comparable.

In view of the similar properties we find in IW Car and the Red Rectangle, we conclude that the expanding gas in IW Car also comes from the disk. From the mass values and outflow kinematics in IW Car, we can derive the typical kinematical time required to form the outflow $(\sim 1000 \mathrm{yr})$ and the average mass-loss rate of the disk. We therefore estimate the disk's future lifetime to be approximately $8000 \mathrm{yr}$. This value is similar to that found in the Red Rectangle, probably longer than that estimated for 89 Her and shorter than for AC Her (Bujarrabal et al. 2016). These lifetime values are comparable to or longer than the expected post-AGB/preplanetary lifetimes of these objects, and the disks could even survive in their (probable) planetary nebula phase. We conclude that the coexistence of rotating equatorial disks and gas in expansion extracted from it is probably a systematic property of this class of post-AGB nebulae and that these are long-living structures.

Acknowledgements. ALMA is a partnership of ESO (representing its member states), NSF (USA) and NINS (Japan), together with NRC (Canada) and NSC and ASIAA (Taiwan), in cooperation with the Republic of Chile. The Joint ALMA Observatory is operated by ESO, AUI/NRAO and NAOJ. We made use of the ALMA dataset ADS/JAO.ALMA\#2013.1.00338.S. This work has been supported by the Spanish MICINN, program CONSOLIDER INGENIO 2010 (grant "ASTROMOL" CSD2009-00038), by the Spanish MINECO (grants AYA2012-32032 and FIS2012-32096), and by the European Research Council (ERC Grant 610256: NANOCOSMOS).

\section{References}

Balick, B., \& Frank, A. 2002, ARA\&A, 40, 439

Bujarrabal, V., van Winckel, H., Neri, R., et al. 2007, A\&A, 468, L45 Bujarrabal, V., Alcolea, J., Van Winckel, H., Santander-García, M., \& Castro-Carrizo, A. 2013a, A\&A, 557, A104

Bujarrabal, V., Alcolea, J., Van Winckel, H., et al. 2013b, A\&A, 557, L11 Bujarrabal, V., Castro-Carrizo, A., Alcolea, J., \& Van Winckel, H. 2015, A\&A, 575, L7

Bujarrabal, V., Castro-Carrizo, A., Alcolea, J., et al. 2016, A\&A, 593, A92 De Marco, O. 2014, Asymmetrical Planetary Nebulae VI Conference, 122 de Ruyter, S., van Winckel, H., Maas, T., et al. 2006, A\&A, 448, 641 Gezer, I., Van Winckel, H., Bozkurt, Z., et al. 2015, MNRAS, 453, 133 Giridhar, S., Rao, N. K., \& Lambert, D. L. 1994, ApJ, 437, 476

Kiss, L. L., Derekas, A., Szabó, G. M., Bedding, T. R., \& Szabados, L. 2007, MNRAS, 375, 1338

Soker, N. 2001, ApJ, 558, 157

Sánchez Contreras, C., Sahai, R., \& Gil de Paz, A. 2002, ApJ, 578, 269

Van Winckel, H. 2003, ARA\&A, 41, 391

Van Winckel, H., Lloyd Evans, T., Briquet, M., et al. 2009, A\&A, 505, 1221 


\section{Appendix A: Further discussion on our simple modeling}

\section{A.1. Model description}

The determination of the structure and dynamics of the nebula around IW Car is difficult because of the lack of information on this source, in particular on the main properties of the nebula. For that reason, we have chosen a simple modeling with few free parameters. See a representation of out model nebula in Fig. 6 and our model predictions in Figs. 2 and A.1 and A.2. The model nebula is in some way a compromise between those we used to describe the observations of 89 Her and the Red Rectangle (Bujarrabal et al. 2007, 2016).

In the rotating equatorial disk, we assume a purely Keplerian rotation velocity in the central biconical region (Fig. 6), closer than $R_{\mathrm{K}}=2 \times 10^{15} \mathrm{~cm}$, with $V_{\mathrm{rot}}\left(R_{\mathrm{K}}=2.5 \mathrm{~km} \mathrm{~s}^{-1}\right)$. In outer parts, we assume that there is also expansion at $3 \mathrm{~km} \mathrm{~s}^{-1}$, superposed to rotation, whose modulus in this case decreases inversely proportional to the distance (following the law of angular momentum conservation; the same was found for the outer part of the disk of the Red Rectangle, which has been studied in much more detail). The density of the disk is assumed to vary with the distance to the star following a simple potential law, $n \propto r^{-2.7}$ with a value of $4 \times 10^{5} \mathrm{~cm}^{-3}$ at half distance and $4 \times 10^{6} \mathrm{~cm}^{-3}$ at the point where the velocity changes to purely Keplerian, $R_{\mathrm{K}}$. The temperature varies proportionally to $r^{-1.5}$, with a temperature of $T=200 \mathrm{~K}$ at $R_{\mathrm{K}}$.

In the hourglass-like outflowing component, we assume radial velocity, with a modulus depending on the distances to the equator $h$ and to the axis $p$ (varying proportionally to $h$ and linearly with $p_{\text {out }}-p$, where $p_{\text {out }}$ is the maximum value of $p$ for a given value of $h$ ); see Fig. 6 . This law is similar to that found for the Red Rectangle. The temperature is assumed to vary again with potential laws depending on the distance to the center: $T(r) \propto r^{-0.5}$, and a typical $T\left(10^{16} \mathrm{~cm}\right)=85 \mathrm{~K}$. At $2 \times 10^{15} \mathrm{~cm}$, high temperatures of approximately $200 \mathrm{~K}$ are attained. The fitting is improved if we assume a more complex variation for the outflow density, depending again on $h$ and $p$; we adopted a law proportional to $h^{-0.5}$ and linear with $p_{\text {out }}-p$. Density values range typically between $\sim 10^{4}$ and $10^{5} \mathrm{~cm}^{-3}$, see Fig. 6 .

\section{A.2. Uncertainties in the model parameters}

Some parameters describing the structure and dynamics show significant uncertainties due to the lack of data on this nebula. The disk width is not resolved, so its structure is uncertain. However, its diameter is accurate within $\sim 20 \%$, given by the extent in the $p-v$ cuts. Variations of this parameter would yield inversely proportional variations of the gas density, but not of its total mass, which must be kept to explain the ${ }^{13} \mathrm{CO}$ measured intensities (see below).

The disk dynamics also show uncertainties. In particular, the point at which the rotational velocity law changes is difficult to determine. The outflow velocity field in the disk is also not easy to describe, but the presence of two regimes is necessary, because otherwise the predicted position-velocity cuts are significantly different from the observational data. See an example in Fig. A.3 of predictions in which a purely Keplerian field is assumed. The outflow velocity modulus is constant and equal to $3 \mathrm{~km} \mathrm{~s}^{-1}$, it probably represents an order of magnitude of the departures from a Keplerian law we must introduce to avoid these problems, more than a well defined velocity field determined from model fitting. Due to the poor information on this

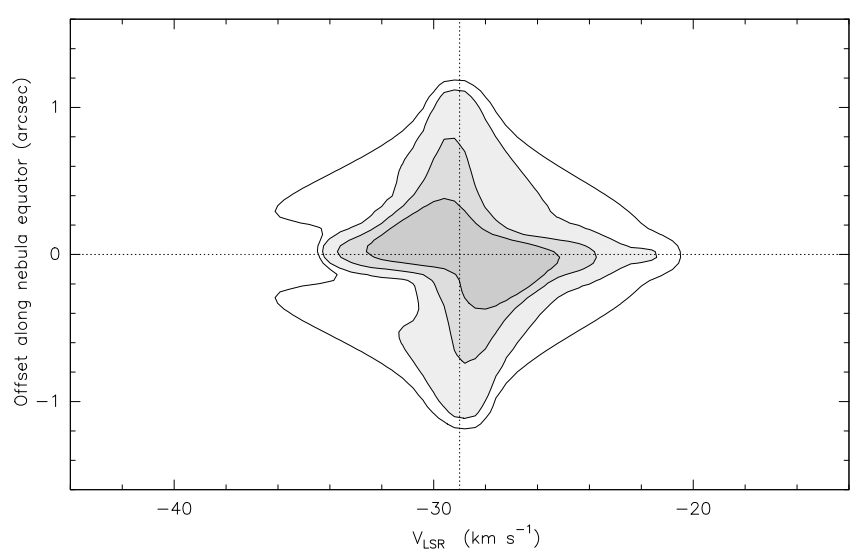

Fig. A.1. Position-velocity diagrams predicted from our simple model for the ${ }^{12} \mathrm{CO} J=3-2$ emission in IW Car along the equatorial direction, to be compared with Fig. 4; contours and the rest of the imaging parameters are the same as in that figure.

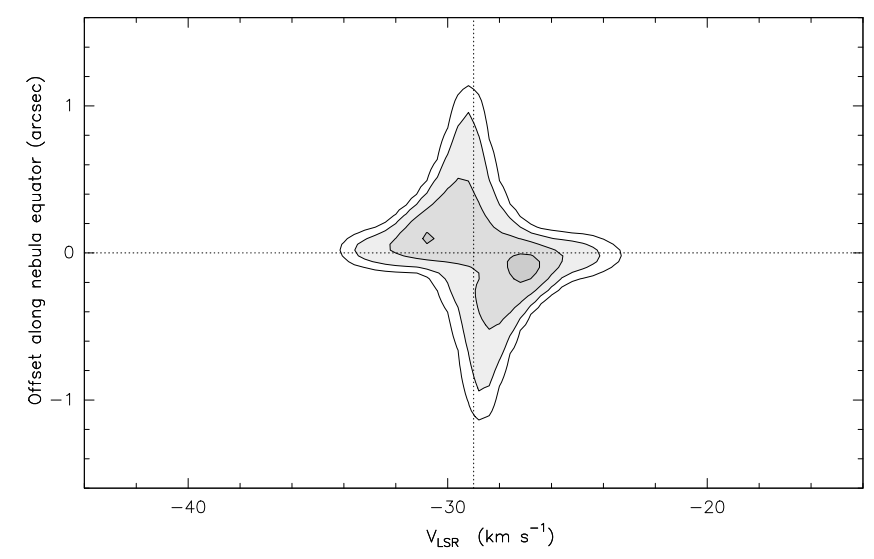

Fig. A.2. As for Fig. A.1 but for ${ }^{13} \mathrm{CO} J=3-2$. Synthetic positionvelocity diagram along the equatorial direction, to be compared with Fig. 5.

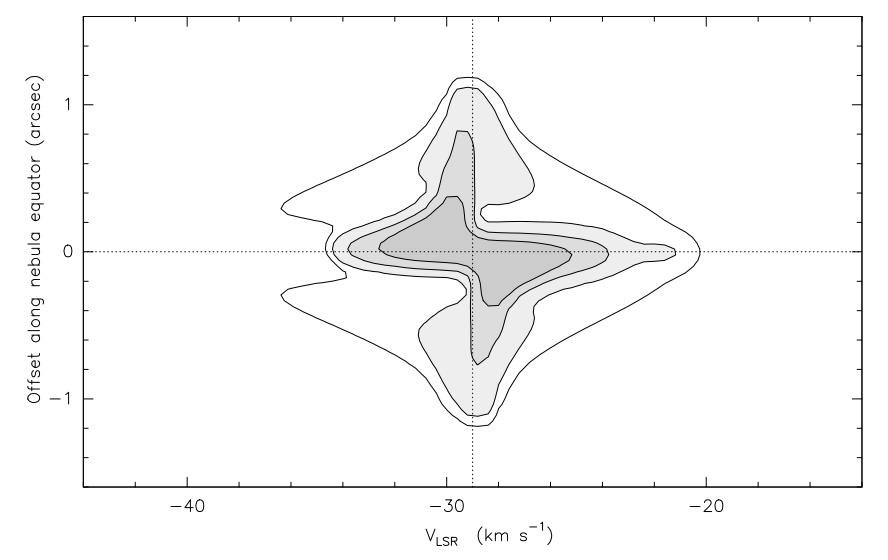

Fig. A.3. As for Fig. A.1 but assuming that the outer disk shows no expansion velocity. The rest of the model parameters are kept the same. The predicted position-velocity field is significantly different from the observations (Fig. 4).

parameter and its meaning, we did not explore possible complex laws, though we recognize that we probably followed an overly simple description. However, the rotation velocity is better constrained because we can identify it in the position-velocity diagrams (see Sect. 3.1). 
The main properties of the hourglass-like component are also difficult to study in detail from the existing data. We assumed radial expansion, one of the simplest laws for the velocity. Radial expansion is a simple definition and the expected case if launching takes place along the force lines of a (locally) radial magnetic field. A linear variation of the absolute value is in general expected if most accelerations take place at the beginning of the process; in our case, variation depending on the latitude and $p_{\text {out }}-p$ (as for the density, see Appendix A.1) leads to somewhat better predictions than dependence on the distance to the center. We can exclude some other simple cases, such as constant velocity modulus, expansion in the axial direction, and expansion parallel to the equator, for which the predictions do not match the observations. However, other velocity laws, such as that found for the outflowing gas in Red Rectangle by Bujarrabal et al. (2016), which is more complex, would lead to results compatible with the data (although involving a higher number of parameters). We stress that the launching mechanism itself is obviously very uncertain and its possible nature is a weak argument to support any velocity field; a theoretical discussion on such processes is beyond the scope of this letter. The total size, general shape, and overall velocity of the outflowing gas are however relatively well constrained, since they are given by the observed structure at several $\mathrm{km} \mathrm{s}^{-1}$ from the central velocity and the total velocity extent of the emission.

As mentioned in Sect. 3.1, the assumption of LTE level population simplifies a lot our analysis. Nevertheless, we must keep in mind that the rotational temperatures we use in our calculations may only represent $J$-averaged excitation temperatures and not true kinetic temperatures. Since high- $J$ levels can obviously be less populated in reality than for LTE, these rotational temperatures are probably a lower limit to the actual ones in the outflowing, relatively diffuse gas.

Another consequence of the assumption of thermalized level populations is that it is not possible to distinguish the effects of the density and the relative $\mathrm{CO}$ abundances, $X\left({ }^{12} \mathrm{CO}\right.$, $\left.{ }^{13} \mathrm{CO}\right)$. Fortunately, $X$ seems relatively well constrained in the best studied objects, particularly $X\left({ }^{13} \mathrm{CO}\right)$, which is the basic parameter to determine the total density and mass because of the lower opacity of ${ }^{13} \mathrm{CO}$ lines. Our previous works always yielded $X\left({ }^{13} \mathrm{CO}\right)$ ranging between $10^{-5}$ and $2 \times 10^{-5}$, both for post-AGB disks and for young PNe in general. We will adopt $X\left({ }^{13} \mathrm{CO}\right) \sim 2 \times 10^{-5}$ to ease the comparison with previous results on this object (Bujarrabal et al. 2013a). In order to match the relatively low ${ }^{12} \mathrm{CO} /{ }^{13} \mathrm{CO}$ intensity ratio, we need a relatively low abundance ratio. We adopt $X\left({ }^{12} \mathrm{CO}\right) \sim 10^{-4}$; similar low ratios are often found in similar objects. ${ }^{13} \mathrm{CO} J=3-2$ is weaker than ${ }^{12} \mathrm{CO} J=3-2$, confirming that the ${ }^{13} \mathrm{CO}$ emission is not very opaque. Our model yields typical values of the optical depth $\tau\left({ }^{12} \mathrm{CO} J=3-2\right) \sim 3$ in the disk (for the representative velocities), while we typically find $\tau\left({ }^{12} \mathrm{CO} J=3-2\right) \lesssim$ 0.2 in the outflow emission. For ${ }^{13} \mathrm{CO} J=3-2$, we find optical depths in the densest parts of the disk $\tau\left({ }^{13} \mathrm{CO} J=3-2\right) \sim$ 1. However, $\tau\left({ }^{13} \mathrm{CO} J=3-2\right) \sim 0.3$ in most of the disk, and it is smaller than 0.1 in most of the lines of sight intersecting the hourglass-like component. Therefore, the determination of the density and mass of the densest parts of the disk remains uncertain, but, except for this case, the mass determination is relatively accurate, provided that we reproduce the total ${ }^{13} \mathrm{CO}$ intensity. The value of the total mass is mainly affected by the assumed $X\left({ }^{13} \mathrm{CO}\right)$ value and depends slightly on other parameters such as the geometry and kinematics.

\section{A.3. Comparison of the predictions with the observational data}

Figure 2 shows the observational and synthetic maps per velocity channel and Figs. A.1 and A.2 show the predicted positionvelocity diagrams along the rotating disk. The model reproduces most of the observed features, but some problems are still present. The major one concerns the faint extended emission, mostly at moderate velocities further than approximately $2 \mathrm{~km} \mathrm{~s}^{-1}$ from the central velocity. The predicted features are always somewhat wider. This is very probably due to the significant loss of flux in the interferometric observation (Sect. 2). This relatively extended and weak emission is expected to be particularly affected by the significant instrumental flux loss, which means that we can expect noisier data and a significant overestimate of the predictions. Because of the steep logarithmic scale we use, changes at a level of approximately 1/30 of the peak brightness (our first contour) are very clearly shown in the figures, even if their effect on the total emission is moderate. This issue also appears in the synthetic position-velocity diagrams, at intermediate velocities and at $\sim \pm 0$.' 5 from the center, which show an overly extended faint emission. We have tried to minimize this effect in our modeling, but it cannot disappear without significantly affecting other predictions, like the total velocity extent, which become incompatible with observations.

We also note that the observed emission extends significantly more at less negative LSR velocities; an asymmetry that we do not try to introduce in our model, which assumes symmetry with respect to the equator and axis. Obviously, the actual nebula does not exactly show such symmetries, which is a common issue when modeling post-AGB nebulae.

Another effect of the presence of expanding gas is the weak absorption that appears at approximately $-35 \mathrm{~km} \mathrm{~s}^{-1}$. The absorption is noticeable in the different shapes observed between relatively redshifted and blueshifted features (Figs. 1 and 2) and in the different blue/red sides of the p-v diagram (Figs. 4, A.1), with blueshifted emission systematically weaker. This phenomenon depends on subtle geometrical and velocity coincidences and is very difficult to model. Our code predicts absorption features similar to those observed but, because of the uncertain treatment of this effect, we will not try to fit it. 